\title{
A Quantum field theory of dyons
}

\author{
Kurt Lechner* \\ Dipartimento di Fisica, Università di Padova, and Istituto Nazionale di Fisica Nucleare, sezione \\ di Padova, Via F. Marzolo 8, 35131 Padova, Italy \\ E-mail: 'kurt.lechner@pd.infn.it'
}

ABSTRACT: We construct a classical field theory action which upon quantization via the functional integral approach, gives rise to a consistent Dirac-string independent quantum field theory. The approach entails a systematic derivation of the correlators of all gauge invariant observables, and also of charged dyonic fields. Manifest $S O(2)$-duality invariance and Lorentz invariance are ensured by the PST-approach.

KEYwords: 'Dyons, Quantum field theory, Dualityı.

\section{Introduction}

Each formulation of a quantum field theory of dyons, particles which carry electric and magnetic charge, in four dimensions has to cope with a fundamental problem: there is no natural or consistent classical field theory action to start with. Nevertheless, there exists a consistent quantum field theory [i]i]

The pathologies of the classical field theory action can be traded in several ways. One can renounce to describe the dynamics of the charged matter in terms of scalar or spinor fields [2] one sacrifices Lorentz-invariance introducing in the kinetic term for the gauge fields a constant four-vector $n^{\mu}\left[I_{1}^{1}\right.$. In the first case the quantum field theory can not be based on a functional integral and the identification of the correct field strength is an open problem. In the second case the presence of the vector $n^{\mu}$ obscures the Lorentz-symmetry structure of the theory at the classical level (it breaks it explicitly), and one has to show that the quantum theory is independent of $n^{\mu}$ if the Dirac-Schwinger quantization

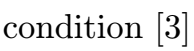

$$
\frac{1}{2}\left(e_{r} g_{s}-e_{s} g_{r}\right)=2 \pi n_{r s}
$$

holds, with $n_{r s}$ integer. Here $e_{r}\left(g_{r}\right)$ is the electric

\footnotetext{
*In collaboration with Pieralberto Marchetti
}

(magnetic) charge of the $r$-th particle ${ }^{1}$. Hence, only a posteriori $n^{\mu}$ acquires the meaning of the direction of the Dirac-string.

The approach we present here relies on a classical field theory action which is manifestly invariant under Lorentz transformations and $S O(2)$ duality, but depends on a fixed external classical vector field $U^{\mu}(x)$. The meaning of this vector field is very simple: the unique integral curve associated to $U^{\mu}$ starting from a point of a particle's trajectory determines the Dirac-string attached to the particle in that instant. The set of all these integral curves determines then a twodimensional surface whose boundary is the trajectory of the particle. This idea can be extended to the case when the currents are not point-like, as in the classical point-particle theory, but continuously distributed, as in the classical field theory.

The consistency check of the construction consists then in showing that the quantum field theory, obtained from the classical field theory action via the traditional functional integral approach, gives rise to correlators which are independent of $U^{\mu}$, if $\left(\begin{array}{l}1 \\ 1\end{array}\right)$ holds. Below we give the

\footnotetext{
${ }^{1}$ We deal here only, with the $S O(2)$-duality invariant theory for which $\left(1_{1}^{1} .1_{1}^{\prime}\right)$ is the appropriate quantization condition. For a discussion of the theory which is only invariant under the discrete duality group $Z_{4}$, see $\left[4_{i}^{i}\right.$. In that case the appropriate quantization condition is Dirac's original one $e_{r} g_{s}=2 \pi n_{r s}$.
} 
outline of the construction, and the proof that the partition function is indeed $U$-independent. For correlators of generic observables, and for further developments and details we refer the

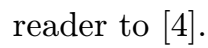

\section{A set of equations of motion}

We start by searching for an appropriate set of equations of motion describing the interaction of a gauge field interacting with a certain number $N$ of dyons, with masses $m_{r}$ and charges $e_{r}^{I} \equiv$ $\left(e_{r},-g_{r}\right) ; I=1,2, r=1, \ldots, N$. For simplicity we consider bosonic dyons, described by complex scalar fields $\varphi_{r}$. To implement $S O(2)$-invariance in a manifest way we introduce for the photon a doublet of one-forms $A^{I}$, such that the covariant derivative on the scalars is given by

$$
D_{\mu}(A) \varphi_{r}=\left(\partial_{\mu}+i e_{r}^{I} \varepsilon^{I J} A_{\mu}^{J}\right) \varphi_{r} .
$$

The hodge duals of the total electric and magnetic currents, a doublet of three-forms, can then be written as

$$
J^{I}=\frac{1}{3 !} d x^{\rho} d x^{\nu} d x^{\mu} \varepsilon_{\mu \nu \rho \alpha} \sum_{r} e_{r}^{I} i \bar{\varphi}_{r} D^{\alpha} \varphi_{r}+c . c .
$$

In the language of differential forms current conservation reads then simply

$$
d J^{I}=0
$$

These equations allow in turn to introduce a doublet of two-forms $C^{I}$ satisfying

$$
J^{I}=d C^{I} .
$$

Clearly these forms are determined only modulo exact forms (we suppose here to work in a fourdimensional space-time with trivial topology).

Maxwell's equations, in the presence of magnetic currents, read in this language

$$
\begin{aligned}
d F^{I} & =J^{I} \\
F^{I} & =* \varepsilon^{I J} F^{J},
\end{aligned}
$$

where $*$ indicates the hodge dual and $\varepsilon^{I J}$ is the two-dimensional antisymmetric $S O(2)$-invariant tensor. $F^{2}$ is the standard field strength twoform, and on-shell we have $F^{1}=* F^{2}$. These equations, together with $(\underline{2} \cdot \overline{4}-\overline{4})$, allow finally to relate $F^{I}$ and $C^{I}$ to the vector potentials

$$
F^{I}=d A^{I}+C^{I}
$$

One could now close the dynamics of the system by adding just the covariant Klein-Gordon equation for the matter fields. This is, however, not sufficient because the fields $C^{I}$ are determined only modulo exact forms, see (12.4).

A convenient way to close the system is represented by the introduction of a vector field $U=$ $U^{\mu}(x) \partial_{\mu}$. We will use the same symbol to indicate the associated one-form $U=d x^{\mu} U_{\mu}$, since no confusion should arise. If we indicate with $i_{U}$ the interior product of a form with the vector field $U$ the supplementary condition on $C^{I}$ can be written as

$$
i_{U} C^{I}=0
$$

with the boundary condition that the fields $C^{I}$ vanish as $x$ goes to minus infinity along the integral curves of $U$. It can then be shown that the system $(2.4),(2.6)$ admits a unique solution. For example, if we take a constant vector $U^{\mu}=N^{\mu}$, the unique solution can be written as ${ }^{2}$

$$
C^{I}=\frac{1}{\partial_{N}} i_{N} J^{I}
$$

where $\partial_{N}=N^{\mu} \partial_{\mu}$. The inverse operator $\frac{1}{\partial_{N}}$ has to cope with the above boundary condition and is defined by the Kernel $G(x)=\Theta\left(x_{N}\right) \delta^{3}\left(\vec{x}_{N}^{\perp}\right)$, $\partial_{N} G(x)=\delta^{4}(x)$, where $\vec{x}_{N}^{\perp}$ are the three coordinates orthogonal to $x_{N}=x^{\mu} n_{\mu}$, and $\Theta$ is the step-function.

We collect here the closed system of equations of motion for the fields $A^{I}, C^{I}, \varphi_{r}$ :

$$
\begin{aligned}
\left(D^{\mu} D_{\mu}+m_{r}^{2}\right) \varphi_{r} & =0 \\
F^{I} & =* \varepsilon^{I J} F^{J} \\
d C^{I} & =J^{I} \\
i_{U} C^{I} & =0 .
\end{aligned}
$$

This system is manifestly $S O(2)$ - and Lorentzinvariant, but depends on an external vector field and is therefore inconsistent. Nevertheless, we can write an invariant action which gives rise to this system.

\footnotetext{
${ }^{2}$ Use the identity $i_{N} d+d i_{N}=\partial_{N}$.
} 
Before doing that let us briefly comment on the point-particle version of the above system. In that case the Klein-Gordon equation is substituted by the generalized Lorentz-force law

$$
m_{r} \frac{d u_{r}^{\mu}}{d \tau_{r}}=\left(e_{r I} \varepsilon^{I J} F_{J}^{\mu \nu}\right)\left(x_{r}\right) u_{r \nu}
$$

and the three-forms $J^{I}$ become a sum of $\delta$-functions along the particle's trajectories; more precisely, we have $J^{I} \rightarrow \mathcal{J}^{I}=\sum_{r} e_{r}^{I} \mathcal{J}_{r}$, where the threeforms $\mathcal{J}_{r}$ are the Poincarè-duals in the space of distributional forms ( $p$-"currents") of the closed particle's trajectories $\gamma_{r}$. Such $p$-forms, which are $\delta$-functions on a $(D-p)$-dimensional submanifold, are called integer forms [ij]]. An important property of such forms is that the integral over all space of the product of two of them is always an integer - hence the name - counting the intersection points of the two manifolds.

The remaining equations remain the same. In particular the solution for the forms $C^{I}$ becomes now $C^{I}=\sum_{r} e_{r}^{I} C_{r}$, where the two-forms $C_{r}$, with $d C_{r}=\mathcal{J}_{r}$, are $\delta$-functions on twodimensional surfaces whose boundaries are the trajectories $\gamma_{r}$. The $r$-th surface is composed of the integral curves of $U$ which start from the points of the trajectory $\gamma_{r}$. The boundary condition introduced above lets the integral curves just end on the trajectories. Therefore, in this case the forms $C_{r}$ represent precisely the Dirac-string "evolving" in time. The Dirac-strings do not really "evolve" since they are completely fixed by the currents, once one has chosen a vector field $U$.

If the currents are continuously distributed, as in the field theory, also the forms $C_{r}$ are spread out, but they are again uniquely determined by the above equations.

\section{Classical field theory action}

We write now an action which gives rise to the system (2. dinary covariant Klein-Gordon action. For the pseudo self-duality equation of motion for Maxwell's fields we employ the PST-approach [i, troduces a scalar auxiliary field $a$ and the oneform

$$
v=\frac{d a}{\sqrt{-\partial_{\rho} a \partial^{\rho} a}} \equiv d x^{\mu} v_{\mu} .
$$

The PST-action can then be written as the integral of a four-form,

$$
S_{0}[A, C, a]=\frac{1}{2} \int F^{I} \mathcal{P}(v)^{I J} F^{J}+d A^{I} \varepsilon^{I J} C^{J} .
$$

$\mathcal{P}(v)$ is a symmetric operator which acts in the space of two-forms and on the $S O(2)$-indices as

$$
\mathcal{P}^{I J}(v)=v i_{v} * \delta^{I J}+\left(v i_{v}-\frac{1}{2}\right) \varepsilon^{I J} .
$$

We remember that the PST-symmetries ensure that $a$ is non propagating and that the (gaugefixed) equations of motion for $A^{I}$ are indeed (2.81).

The equations $(2.91)$ and $(2.10)$ are implied by a convenient set of (auxiliary) Lagrange multiplier fields. We introduce a doublet of auxiliary one-forms $\tilde{A}^{I}$ and a doublet of auxiliary two-forms $\tilde{C}^{I}$. The action, which depends also on the fixed vector $U$, can then be written as $\left(\phi \equiv\left(A, C, \tilde{A}, \tilde{C}, \varphi_{r}, a\right)\right.$

$$
\begin{aligned}
S_{U}[\phi]= & S_{0}-\sum_{r} \int d^{4} x \bar{\varphi}_{r}\left(D_{r}^{2}(\tilde{A})+m_{r}^{2}\right) \varphi_{r} \\
& +\int\left(\tilde{A}^{I} \varepsilon^{I J} d C^{J}-\frac{1}{2} \tilde{C}^{I} U i_{U} \varepsilon^{I J} C^{J}\right) .
\end{aligned}
$$

Notice that in the covariant derivative for the scalars we replaced $A^{I}$ with $\tilde{A}^{I}$. So, variation with respect to $\tilde{A}^{I}$ gives (2.29), while variation with respect to $\tilde{C}^{I}$ gives $\left(2.10_{1}^{\prime}\right)$. As shown in

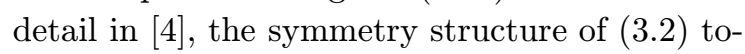
gether with the equations of motion for $C^{I}$ determine also the Lagrange multiplier fields,

$$
\tilde{A}^{I}=A^{I}, \tilde{C}^{I}=C^{I} .
$$

So there are no unwanted propagating degrees of freedom, and the action (3.24) reproduces (12.7)$(12 . \overline{10})$.

\section{A representation for the partition function}

The quantum field theory can be based in a traditional manner on the action (3.2) through the functional integral approach. The correlation functions of (gauge)-invariant operators are expressed as

$$
\left\langle T O_{1} \cdots O_{n}\right\rangle=\int\{\mathcal{D} \phi\} e^{i S_{U}[\phi]} O_{1} \cdots O_{n},
$$


where in the functional integral measure gauge fixings of the relevant invariances, PST-symmetries and $U(1)$-symmetries are understood, and the integration over the fields $C^{I}$ inherits the boundary condition along $U$ from the classical field theory. As they stand, these correlation functions depend on $U$. The fundamental point is that one can show that the correlation functions of all invariant operators are independent of $U\left[\begin{array}{l}{[4} \\ 4\end{array}\right]$, if the Dirac-Schwinger quantization condition (1.1.) holds. Here we limit ourselves to show that the partition function is invariant, in that the strategy followed in the proof extends rather directly to the case of generic observables. To do this, we need a convenient representation for the partition function. The rest of this section is devoted to derive this representation, see (4.6i).

We begin by performing the functional integration over the fields $A$ and $a$ which appear only in the PST-action. Since $a$ is auxiliary it can be gauge-fixed to an arbitrary function $a_{0}(x)$ by inserting a $\delta$-function $\delta\left(a-a_{0}\right)$. Clearly, the partition function has to be independent of $a_{0}$. The integration over the fields $A^{I}$ is gaussian, but one has to carefully fix the PST- and $U(1)-$ symmetries. The resulting effective action $\Gamma[C]$, which depends only on $C^{I}$, can be computed to be

$$
\begin{aligned}
e^{i \Gamma[C]} & \equiv \int\{\mathcal{D} A\}\{\mathcal{D} a\} e^{i S_{0}[A, C, a]} \\
\Gamma[C] & =-\frac{1}{2} \int\left(d C^{I} \frac{*}{\square} d C^{I}-d C^{I} \frac{\varepsilon^{I J}}{\square} * d * C^{J}\right)
\end{aligned}
$$

Notice that it depends on the currents not only through $J^{I}=d C^{I}$, but also through the "Diracstrings" $C^{I}$ in the second term.

The integration over the scalar fields amounts to the evaluation of the corresponding covariant Klein-Gordon determinants. These determinants can be represented in a standard way as Feynman path-integrals over classical parti-

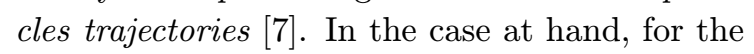
product of all the determinants the relevant representation can be written in a compact way as

$$
\begin{aligned}
& \prod_{r=1}^{N} \operatorname{det}^{-1}\left(-i\left(D_{r}^{2}(\tilde{A})+m_{r}^{2}\right)\right)= \\
& \int\{\mathcal{D} \gamma\} e^{-i \sum_{r=1}^{N} \oint_{\gamma_{r}} \tilde{A}^{I} \varepsilon^{I J} e_{r}^{J}} .
\end{aligned}
$$

Here $\{\mathcal{D} \gamma\}$ indicates a (complicated) measure over the closed particle's trajectories $\gamma_{r}$ whose details are, however, not needed for our purposes. The essential feature of this representation is that it retrieves in the quantum field theory a classical point particle nature. This point is essential for what concerns the proof of Dirac-string independence, as we will see in a moment. The exponent in this representation can also be rewritten as the integral of a four-form

$$
\sum_{r=1}^{N} \oint_{\gamma_{r}} \tilde{A}^{I} \varepsilon^{I J} e_{r}^{J}=\int \tilde{A}^{I} \varepsilon^{I J} \mathcal{J}^{J}
$$

where $\mathcal{J}^{I}=\sum_{r} e_{r}^{I} \mathcal{J}_{r}$ and the three-forms $\mathcal{J}_{r}$ are just $\delta$-functions on the curves $\gamma_{r}$, i.e. their Poincarè-duals. Since these curves are closed we have $d \mathcal{J}^{I}=0=d \mathcal{J}_{r}$.

Integration over $\tilde{C}^{I}$ gives the $\delta$-functions $\delta\left(i_{U} C\right)$ and, suppressing the $S O(2)$-indices, the partition function can be written as

$$
\begin{aligned}
Z & =\int\{\mathcal{D} \phi\} e^{i S_{U}[\phi]} \\
& =\int\{\mathcal{D} \gamma \mathcal{D} C \mathcal{D} \tilde{A}\} \delta\left(i_{U} C\right) e^{i \Gamma[C]+i \int \tilde{A} \varepsilon(d C-\mathcal{J})} \\
& =\int\{\mathcal{D} \gamma \mathcal{D} C\} \delta\left(i_{U} C\right) \delta(d C-\mathcal{J}) e^{i \Gamma[C]}
\end{aligned}
$$

The $\delta$-functions restrict the variables $C^{I}$ to

$$
\begin{aligned}
d C^{I} & =\mathcal{J}^{I} \\
i_{U} C^{I} & =0
\end{aligned}
$$

which, are precisely the classical field theory equations for these fields, but now the currents correspond to classical point-like particles. Taking the boundary conditions into account, we know that these equations admit a unique solution,

$$
C^{I}(U)=\sum_{r} e_{r}^{I} C_{r}(U),
$$

which depends on $U^{\mu}$ and on the classical currents $\mathcal{J}_{r}$. This allows eventually to write the partition function as

$$
Z=\int\{\mathcal{D} \gamma\} e^{i \Gamma[C(U)]} .
$$

Using this representation for $Z$ we can now show that it is $U$-independent. 


\section{Dirac-anomaly and Dirac-string independence}

We recall that the two-forms $C_{r}(U)$ are $\delta$-functions on the surfaces made out of integral curves of $U$ ending on the trajectories $\gamma_{r} . Z$ depends on $U$ only through $C_{r}(U)$. Under a (finite) change of $U^{\mu} \rightarrow U^{\prime \mu}$ the two-forms $C_{r}(U)$ change by an exact form

$$
C_{r}\left(U^{\prime}\right)=C_{r}(U)+d H_{r}\left(U^{\prime}, U\right),
$$

as can be seen from $\left(\left(_{-1}^{-1} \overline{3}\right)\right.$, (the currents $\mathcal{J}$ remain clearly fixed). The important point is, however, that the one-forms $H_{r}$ are integer forms, they are $\delta$-functions on a three-manifold, which is bounded by the two two-dimensional surfaces associated to $C_{r}(U)$ and $C_{r}\left(U^{\prime}\right)$. Therefore, changing $U$ amounts precisely to change the Diracstring attached to the particle in each point: it moves from an integral curve of $U$ to an integral curve of $U^{\prime}$. For the total $C^{I}$ we have then

$$
C^{I I}=C^{I}+d H^{I}, \quad H^{I} \equiv \sum_{r} e_{r}^{I} H_{r}
$$

We can now evaluate the "Dirac-anomaly", i.e. the variation of the effective action $\Gamma[C(U)]$, see (4. by an exact differential, only the second term in (4.1.) contributes and one gets for the Diracanomaly $^{3}$

$$
\begin{aligned}
\mathcal{A}_{D} & \equiv \Gamma\left[C\left(U^{\prime}\right)\right]-\Gamma[C(U)] \\
& =\frac{1}{2} \int \mathcal{J}^{I} \varepsilon^{I J} H^{I} \\
& =\frac{1}{2} \sum_{r, s}\left(e_{r}^{I} \varepsilon^{I J} e_{s}^{J}\right) \int \mathcal{J}_{r} H_{s} \\
& =2 \pi \sum_{r, s} n_{r s} \int \mathcal{J}_{r} H_{s},
\end{aligned}
$$

where in the last line we used the charge quantization condition (1.1.1). Since the three-forms $\mathcal{J}_{r}$ as well as the one-forms $H_{s}$ are integer forms, also the integrals in the last line are integer, and the Dirac-anomaly becomes an integer multiple of $2 \pi$. Therefore, under a change of $U$ the exponent in (4.6i) changes by an integer multiple of $2 \pi$ and the partition function is $U$-independent.

\footnotetext{
${ }^{3} \mathrm{Use} * d * d+d * d *=\square$
}

\section{Further developments and conclud- ing remarks}

The strategy illustrated above can be generalized to prove Dirac-string independence of the correlators of generic observables. It extends in a straightforward way to the correlators of currents, Wilson loops, and neutral Mandelstamstring observables ("mesons"). The correlators of the electromagnetic field strength $F^{I}=\left(F^{1}, F^{2}\right)$ and of charged operators, instead, present additional problems.

The difficulty with the electromagnetic field strength is related with the fact that, to cope with manifest duality, we have introduced two of them, $F^{1}$ and $F^{2}$. Only if the classical equations of motion (2.8) hold we have the identification $F^{1}=* F^{2}$, but this relation does not hold in the functional integral. This means that the correlators of $F^{1}$ do not coincide with the correlators of $* F^{2}$. This mismatch is solved by the observation that the quantities $F^{I}$ can not represent the electromagnetic field strength off-shell. They are, in fact, gauge invariant, but they are not invariant under the PST-symmetries. We need a couple of two-forms $K^{I}$ which are invariant under the PST-symmetries and which reduce on-shell (i.e. under $(\overline{2} 2.81))$ to the $F^{I}$. Such forms exist, indeed, and they are given by

$$
K^{I}=F^{I}-v i_{v}\left(F^{I}-* \varepsilon^{I J} F^{J}\right) .
$$

The key point is that the fields $K^{I}$ satisfy, moreover, identically the pseudo self-duality relation

$$
K^{I}=* \varepsilon^{I J} K^{J} .
$$

Their correlators solve, therefore, automatically the problem related with the mismatch between $F^{1}$ and $* F^{2}$. It can also be shown that, despite the explicit appearance of the field $a$ in their definition, the correlators of the $K^{I}$ 's are independent of $a_{0}(x)$, the gauge-fixed $a$-field, manifestly Lorentz-invariant and $U$-independent.

The problem regarding the correlators of charged fields is related with the correct definition of the related gauge-invariant charged field operators. The extension of Mandelstam's proposal $[\overline{8}]$ for such field operators to the dyonic case would be 
given by ${ }^{4}$

$$
\phi_{r}\left(x, \gamma_{x}\right)=\varphi_{r}(x) \exp \left(i \int_{\gamma_{x}} e_{r}^{I} \varepsilon^{I J} \tilde{A}^{J}\right)
$$

where $\gamma_{x}$ indicates a path which goes from $x$ to infinity, the Mandelstam-string. The correlators of these operators can indeed be seen to be Dirac-string independent - in the present formulation $U$-independent - but they are plagued by (non-renormalizable) infrared divergences, due to the infinite extension of the Mandelstam string.

An alternative proposal for charged operators, due to Dirac, corresponds to substitute in (6.1.) the "singular" Mandelstam-string with a radially symmetric Coulomb potential, which behaves as $1 / r^{2}$, and cures the infrared divergences. But this time the electric flux is spread out continuously in space, and the correlators depend on the Dirac-string (the Coulomb potential is not an "integer form").

A solution of the problem has been proposed in $[\bar{i} \mid$, starting from Mandelstam's proposal. One replaces the single Mandelstam-string $\gamma_{x}$ with a sum over such strings, weighted by a convenient measure

$$
\Phi_{r}(x)=\varphi_{r}(x) \int\left\{\mathcal{D} \gamma_{x}\right\} \exp \left(i \int_{\gamma_{x}} e_{r}^{I} \varepsilon^{I J} \tilde{A}^{J}\right) .
$$

The corresponding correlators are now Diracstring independent. Moreover, the measure $\left\{\mathcal{D} \gamma_{x}\right\}$ has been constructed (implicitly) in [igin], and there it has also been shown that at large distances, on average, it reproduces the Coulomb potential.

The method presented in this talk applies equally well to fermions; the Feynman path-integral representations for the determinants, like (44.2. are available for spinor fields, too. Also, the introduction of $\vartheta$-angles does not encounter any difficulty. Due to manifest Lorentz-invariance of the PST-approach, it admits also a canonical diffeomorphism invariant coupling to gravity. Dirac-string independence follows in this case from the analogous result in the flat case. This is due to the fact that the Dirac-anomaly $(5.1)$ is a topological invariant, i.e. metric independent.

\footnotetext{
${ }^{4}$ For an explanation of the appearance of the fields $\tilde{A}^{I}$ instead of the fields $A^{I}$ see [4].
}

For the duality properties of the model we refer the reader to $\left[\begin{array}{l}{[4]} \\ -1\end{array}\right]$.

The techniques illustrated in this talk can be also applied to a system of interacting $p$-branes, dual branes, and dyonic branes in a generic $D_{-}$ dimensional space-time [1 [10

The formulation of a quantum field theory for dyons presented here can be seen to be equivalent to previous formulations [1]1, concerns the details which have been worked out in those formulations.

The advantage of the present formulation is constituted by the manifest Lorentz-invariance at each step, by the clear identification of the Dirac-string (represented by the field $U$ ) from the beginning, and by a systematic derivation of the observables which are triggered by the symmetries of the action $(3.2 i)$.

\section{References}

[1] R.A. Brandt, F. Neri and D. Zwanziger, Phys. Rev. D19 4 (1979) 1153.

[2] G. Calucci, R. Iengo and M.T. Vallon, Nucl. Phys. B197 (1982) 93; B211 (1983) 77.

[3] P.A.M. Dirac, Proc. R. Soc. London $\mathbf{A 1 3 3}$ (1931) 60; J. Schwinger, Phys. Rev. D12 10 (1975) 3105.

[4] K. Lechner and P.A. Marchetti, hepth/9906079.

[5] G. de Rham, "Differentiable manifolds. Forms, Currents, Harmonic Forms", Springer-Verlag (1984).

[6] P. Pasti, D. Sorokin and M. Tonin, Phys. Lett. B352 (1995) 59; Phys. Rev. D52R (1995) 4277; N. Berkovits and R. Medina, Phys. Rev. D56 (1997) 6388.

[7] T. Jaroszewicz and P.S. Kurzepa, Ann. Phys. 210 (1991) 255.

[8] S. Mandelstam, Ann. Phys. 19 (1962) 1.

[9] J. Froehlich and P.A. Marchetti, "Gauge invariant charged monopole and dyon fields in gauge theories", hep-th/9812004, to appear in Nucl. Phys. B.

[10] K. Lechner and P.A. Marchetti, in preparation. 